\title{
Asymmetrical Gating with Application on Maneuvering Target Tracking
}

\author{
Farzad Hashemzadeh \\ Faculty of Electrical Engineering, Sarab Islamic Azad University, Sarab 54716-376, Iran \\ Correspondence should be addressed to Farzad Hashemzadeh, farzad.hashemzadeh@ualberta.ca
}

Received 22 November 2011; Revised 6 January 2012; Accepted 26 January 2012

Academic Editor: Anyong Qing

Copyright ( $) 2012$ Farzad Hashemzadeh. This is an open access article distributed under the Creative Commons Attribution License, which permits unrestricted use, distribution, and reproduction in any medium, provided the original work is properly cited.

\begin{abstract}
A new asymmetrical gate with application in target tracking is proposed. Proposed gate has asymmetric shape that has large probability of target detection in the gate and has more advantages compared with elliptical gate. The gate is defined as the region in which the tracked target is expected to exist and just observation vectors in the gate are used as target detection. An analytical method to compute optimal size of gate is proposed and recursive estimation of asymmetric parameters of gate are studied. Comparison between proposed gate and conventional elliptical gate showed the efficiency of the proposed method in maneuvering target tracking applications and simulation results showed the proficiency of the proposed method.
\end{abstract}

\section{Introduction}

Tracking is meant to be the estimation of the true values of specifications of target motion, such as the position and velocity, based on the $n$-dimensional observational vector by the sensors [1-3]. One-dimensional observation vectors are obtained in a direction-finding setup observing just the azimuth angle [4]. Two-dimensional observation vectors are obtained in optical sensors observing the azimuth angle and elevation angle, or in radars observing the range and azimuth angle. Historically, PDA (Probabilistic Data Association) [5], JPDA (Joint PDA) [6], and MHT (Multiple Hypothesis Tracking) [7-9] have drawn attention as target tracking methods in an environment in which false signals from objects other than the target such as clutter exist $[10,11]$. In target tracking in such an environment, just the region is considered for each target in which the target is expected to exist at the next sampling time [12]. This region is called the gate. The observation data within the gate are used for tracking. Various gate shapes are conceivable, such as a rectangle, circle, and ellipse. However, it is not known which shape of gate is optimum. If the gate is enlarged, many observation vectors from clutter or objects other than the target fall within the gate, resulting in some difficulties in target tracking. On the other hand, if the gate is made smaller, there is a danger that the observation data from the target to be tracked may not fall within the gate. It is more desirable that the position of the observation vector detected from the target be closer to the center of the gate compared with location of the false signals in the gate [13]. A method of determining the in-gate probability has been proposed in [10].

In this paper, asymmetrical gaussian distribution is introduced. Cross-surface of asymmetrical gaussian distribution is considered as asymmetrical gate. Parameters of asymmetrical Gaussian distribution should be estimated to estimate parameters of asymmetrical gate. Kalman filter as a standard method to estimate parameters of symmetrical distribution is used to estimate parameters of asymmetrical distribution. Standard deviation of asymmetrical distribution in opposite to the movement direction is estimated using estimation of acceleration in movement direction in centric coordinate system. In this paper, analytical preference of asymmetrical gate compared with the elliptical gate is studied. In this analytical study, volumes of gates are considered as a comparison criteria subjected to the assumption that in-gate probability of distribution for both asymmetric and elliptical gates are equal. 
In the following of this paper, Section 2 introduces asymmetrical gaussian distribution. Section 3 is about estimation of parameters of gate. In Section 4, analytical comparison between asymmetrical gate and elliptical gate is considered. In Section 5, optimal size of gate is calculated and in Section 6, preference of this new method is shown by simulation result.

\section{Asymmetric Gaussian Gate}

Maneuvering target that has nonzero acceleration in direction of movement could be modeled with almost-constantacceleration target motion model in which process and measurement noise on direction of movement have asymmetric distribution and process and measurement noises on orthogonal to the movement direction have gaussian distribution.

In this paper, it is assumed that distribution of location of maneuvering target in the next time is asymmetric gaussian in centric coordinate system. Center of centric coordinate system is in the location of target and directions of axis of it are in the movement direction and orthogonal to the movement direction.

One-dimensional asymmetric Gaussian distribution in direction of movement is illustrated as follows:

$$
f\left(x_{t}\right)= \begin{cases}\frac{1}{k} \exp \left(-\frac{1}{2} \frac{\left(x_{t}-m\right)^{2}}{\sigma_{x_{1}}^{2}}\right) & x_{t}>m, \\ \frac{1}{k} \exp \left(-\frac{1}{2} \frac{\left(x_{t}-m\right)^{2}}{\sigma_{x_{2}}^{2}}\right) & x_{t}<m,\end{cases}
$$

where $k$ is normalization factor and can be calculated as follow:

$$
\begin{aligned}
k & =\int_{-\infty}^{m} \exp \left(-\frac{1}{2} \frac{\left(x_{t}-m\right)^{2}}{\sigma_{x_{1}}^{2}}\right)+\int_{m}^{+\infty} \exp \left(-\frac{1}{2} \frac{\left(x_{t}-m\right)^{2}}{\sigma_{x_{2}}^{2}}\right) \\
& =\frac{\sqrt{2 \pi \sigma_{x_{1}}^{2}}}{2}+\frac{\sqrt{2 \pi \sigma_{x_{2}}^{2}}}{2}=\sqrt{2 \pi}\left(\frac{\sigma_{x_{1}}+\sigma_{x_{2}}}{2}\right) .
\end{aligned}
$$

In (2), $\sigma_{x_{1}}$ and $\sigma_{x_{2}}$ are standard deviation of distribution of $x_{t}$ in positive and negative directions, respectively, and $m$ is median of distribution of $x_{t}$.

Considering (2) and (1), one-dimensional asymmetric gaussian distribution of $x_{t}$ can be achieved as follows:

$$
\begin{aligned}
& f\left(x_{t}\right) \\
& \quad= \begin{cases}\frac{1}{\sqrt{2 \pi}\left(\left(\sigma_{x_{1}}+\sigma_{x_{2}}\right) / 2\right)} \exp \left(-\frac{1}{2} \frac{\left(x_{t}-m\right)^{2}}{\sigma_{x_{1}}^{2}}\right) & x_{t}>m, \\
\frac{1}{\sqrt{2 \pi}\left(\left(\sigma_{x_{1}}+\sigma_{x_{2}}\right) / 2\right)} \exp \left(-\frac{1}{2} \frac{\left(x_{t}-m\right)^{2}}{\sigma_{x_{2}}^{2}}\right) & x_{t}<m .\end{cases}
\end{aligned}
$$

For $x_{t}>m, f\left(x_{t}\right)$ is same as typical Gaussian distribution with standard deviation $\sigma_{x_{1}}$ and for $x_{t}<m$, it is the same as typical Gaussian distribution with standard deviation $\sigma_{x_{2}}$.

If $\sigma_{x_{1}}=\sigma_{x_{2}},(3)$ is standard Gaussian distribution and if $\sigma_{x_{1}} \neq \sigma_{x_{2}},(3)$ is asymmetrical Gaussian distribution. It means

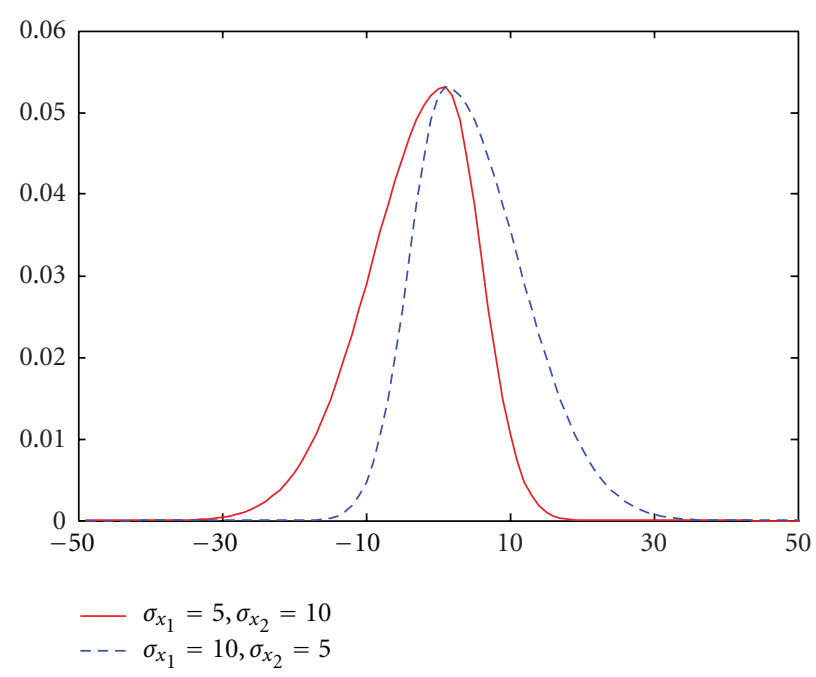

Figure 1: Distribution of $f\left(x_{t}\right)$ with zero mean and two variant variances.

that distribution is not symmetrical around median $m$, and dampings of distribution are different around of the median.

In Figure 1, distribution of $f\left(x_{t}\right)$ with zero median and two different standard deviations is shown.

It is assumed that Probability Distribution Function (PDF) of target in orthogonal to the direction of movement is zero mean gaussian with standard deviation as follows:

$$
f\left(y_{t}\right)=\frac{1}{2 \pi \sigma_{y}} \exp \left(-\frac{1}{2} \frac{\left(y_{t}\right)^{2}}{\sigma_{y}^{2}}\right) .
$$

In this paper, $z_{t}=\left[\begin{array}{ll}x_{t} & y_{t}\end{array}\right]^{T}$ is defined in centric coordinate system, in which $x_{t}$ and $y_{t}$ are measured values of target in centric coordinate system.

Probability distribution of $z_{t}$ in centric coordinate system is illustrated as follows:

$$
\begin{aligned}
& f\left(x_{t}, y_{t}\right) \\
& \quad= \begin{cases}\frac{1}{k} \exp \left(-\frac{1}{2}\left(z_{t}-m_{z}\right) \Sigma_{1}^{-1}\left(z_{t}-m_{z}\right)^{T}\right) & x_{t}>m, \\
\frac{1}{k} \exp \left(-\frac{1}{2}\left(z_{t}-m_{z}\right) \Sigma_{2}^{-1}\left(z_{t}-m_{z}\right)^{T}\right) & x_{t}<m,\end{cases}
\end{aligned}
$$

where

$$
m_{z}=\left[\begin{array}{ll}
m & 0
\end{array}\right], \quad \Sigma_{1}=\left[\begin{array}{cc}
\sigma_{x_{1}}^{2} & 0 \\
0 & \sigma_{y}^{2}
\end{array}\right], \quad \Sigma_{2}=\left[\begin{array}{cc}
\sigma_{x_{2}}^{2} & 0 \\
0 & \sigma_{y}^{2}
\end{array}\right] .
$$


In (5), $k$ is normalization factor and can be calculated as

$$
\begin{aligned}
k= & \int_{-\infty}^{m} \int_{-\infty}^{+\infty} \exp \left(-\frac{1}{2}\left(\frac{(x-m)^{2}}{\sigma_{x_{2}}^{2}}+\frac{y^{2}}{\sigma_{y}^{2}}\right)\right) d y d x \\
& +\int_{m}^{+\infty} \int_{-\infty}^{+\infty} \exp \left(-\frac{1}{2}\left(\frac{(x-m)^{2}}{\sigma_{x_{1}}^{2}}+\frac{y^{2}}{\sigma_{y}^{2}}\right)\right) d y d x \\
= & 2 \pi \frac{\sigma_{x_{1}} \sigma_{y}}{2}+2 \pi \frac{\sigma_{x_{2}} \sigma_{y}}{2}=2 \pi \sigma_{y}\left(\frac{\sigma_{x_{1}}+\sigma_{x_{2}}}{2}\right) \\
= & \pi\left|\Sigma_{1}^{1 / 2}+\Sigma_{2}^{1 / 2}\right| .
\end{aligned}
$$

Considering (5) and (7), probability distribution of $f\left(x_{t}, y_{t}\right)$ in centric coordinate system can be achieved as follows:

$$
\begin{aligned}
& f\left(x_{t}, y_{t}\right) \\
& =\left\{\begin{array}{l}
\frac{1}{2 \pi \sigma_{y}\left(\left(\sigma_{x_{1}}+\sigma_{x_{2}}\right) / 2\right)} \\
\quad \times \exp \left(-\frac{1}{2}\left(z_{t}-m_{z}\right) \Sigma_{1}^{-1}\left(z_{t}-m_{z}\right)^{T}\right) \quad x_{t}>m, \\
\frac{1}{2 \pi \sigma_{y}\left(\left(\sigma_{x_{1}}+\sigma_{x_{2}}\right) / 2\right)} \\
\quad \times \exp \left(-\frac{1}{2}\left(z_{t}-m_{z}\right) \Sigma_{2}^{-1}\left(z_{t}-m_{z}\right)^{T}\right) \quad x_{t}<m .
\end{array}\right.
\end{aligned}
$$

Probability distributions of $f\left(x_{t}, y_{t}\right)$ in (8) with surfaces of distribution are illustrated in Figure 2.

As it can be shown from Figures 2(a) and 2(b), with variation of ratio of $\sigma_{x_{1}}$ and $\sigma_{x_{2}}$, it is easy to stretch distribution surface in movement direction or opposite of movement direction. Application of tuning of variances is that, for maneuvering targets that have positive or negative accelerations on direction of movement, it is possible to modify surface of distribution, optimally.

If maneuvering target has positive acceleration in direction of movement, it is better to select $\sigma_{x_{1}}>\sigma_{x_{2}}$ to stretch gate in positive direction of movement as Figure 2(a) and if maneuvering target has been negative acceleration in direction of movement, it is better to select $\sigma_{x_{1}}<\sigma_{x_{2}}$ to stretch gate in the negative direction of movement as Figure 2(b). Therefore, with appropriate choosing of $\sigma_{x_{1}}$ and $\sigma_{x_{2}}$, it is possible to stretch the gate in direction that probability of target detection inside of the gate increases, whereas volume of gate remains constant.

\section{Gate's Parameters Estimation}

In this section, parameter estimation of target position distribution is considered. If process and measurement noise have Gaussian distribution, Kalman filter would be optimal recursive method to estimate parameters of distribution. Therefore, typical elliptical gate would be optimal tracking gate in such cases [14].

In this paper, maneuvering target with positive or negative acceleration in movement direction is considered.
Kalman filter as a standard recursively estimation method is used to recursively estimate of asymmetrical gate's parameters.

Let us assumed that parameters of asymmetrical gate on time $t-1$ are $m_{t-1}, \sigma_{x_{1}, t-1}, \sigma_{x_{2}, t-1}, \sigma_{y, t-1}, \theta_{t-1}$, where $m_{t-1}$ is center of gate, $\sigma_{x_{1}, t-1}, \sigma_{x_{2}, t-1}$ shows asymmetric stretching of gate in the direction of movement, $\sigma_{y, t-1}$ is the stretching parameter of gate in orthogonal to the movement direction and $\theta_{t-1}$ is rotation angle of centric coordinate system. The goal is to estimate parameters of gate at time $t$.

Using Kalman filter as a standard method in parameter estimation, median and covariance matrixes could be calculated as follows:

$$
\begin{gathered}
m_{t \mid t-1}=A m_{t-1 \mid t-1}, \\
\Sigma_{t \mid t-1}=A \Sigma_{t-1 \mid t-1} A^{T}+Q, \\
m_{t \mid t}=m_{t \mid t-1}+K_{t}\left(z_{t}-C m_{t \mid t-1}\right), \\
\Sigma_{t \mid t}=\Sigma_{t \mid t-1}-K_{t} C \Sigma_{t \mid t-1}, \\
S_{t}=C \Sigma_{t \mid t-1} C^{T}+R, \\
K_{t}=\Sigma_{t \mid t-1} C^{T} S_{t}^{-1} .
\end{gathered}
$$

Typical elliptical tracking gate at time $t$, could be formulated using median $m_{t \mid t}$ and innovation covariance $S_{t}$ as follows:

$$
\left(z_{t}-m_{t \mid t}\right)^{T} S_{t}^{-1}\left(z_{t}-m_{t \mid t}\right) \leq \gamma
$$

where $z$ is the validated measurement and $\gamma$ is a parameter to adjust gate size.

Diagonal terms of innovation covariance $S_{t}$ are diameters of elliptical gate which are standard deviations of gaussian distribution on centric coordinate system. Off-diagonal terms of $S_{t}$ represent rotation angle of centric coordinate system.

Parameters of typical elliptical gate at time $t$ are $\sigma_{x, t}, \sigma_{y, t}$ and $\theta_{t}$

In Figure 3, elliptical gate in $x_{1} o x_{1}$ is represented as $x^{T} \Sigma_{2}^{-1} x=1$ and in $X_{1} O X_{1}$ is represented as $X^{T} \Sigma_{1}^{-1} X=1$, where

$$
\begin{gathered}
\Sigma_{2}=\left[\begin{array}{cc}
\sigma_{x, t}^{2} & 0 \\
0 & \sigma_{y, t}^{2}
\end{array}\right], \quad \Sigma_{1}=S_{t}, \\
X=\left[\begin{array}{ll}
X_{1} & X_{2}
\end{array}\right]^{T}, \quad x=\left[\begin{array}{ll}
x_{1} & x_{2}
\end{array}\right]^{T} .
\end{gathered}
$$

Coordinate systems $x_{1} o x_{1}$ is transferred to the $X_{1} O X_{1}$ using simple transfer matrix $T$ as follows:

$$
X=T x \quad \text { where } T=\left[\begin{array}{cc}
\cos \theta & -\sin \theta \\
\sin \theta & \cos \theta
\end{array}\right] .
$$

In the following, relation between parameters of $\Sigma_{2}$ and $\Sigma_{1}$ are shown:

$$
\begin{gathered}
x^{T} \Sigma_{2}^{-1} x=X^{T} \Sigma_{1}^{-1} X, \\
\Sigma_{2}^{-1}=T^{T} \Sigma_{1}^{-1} T .
\end{gathered}
$$



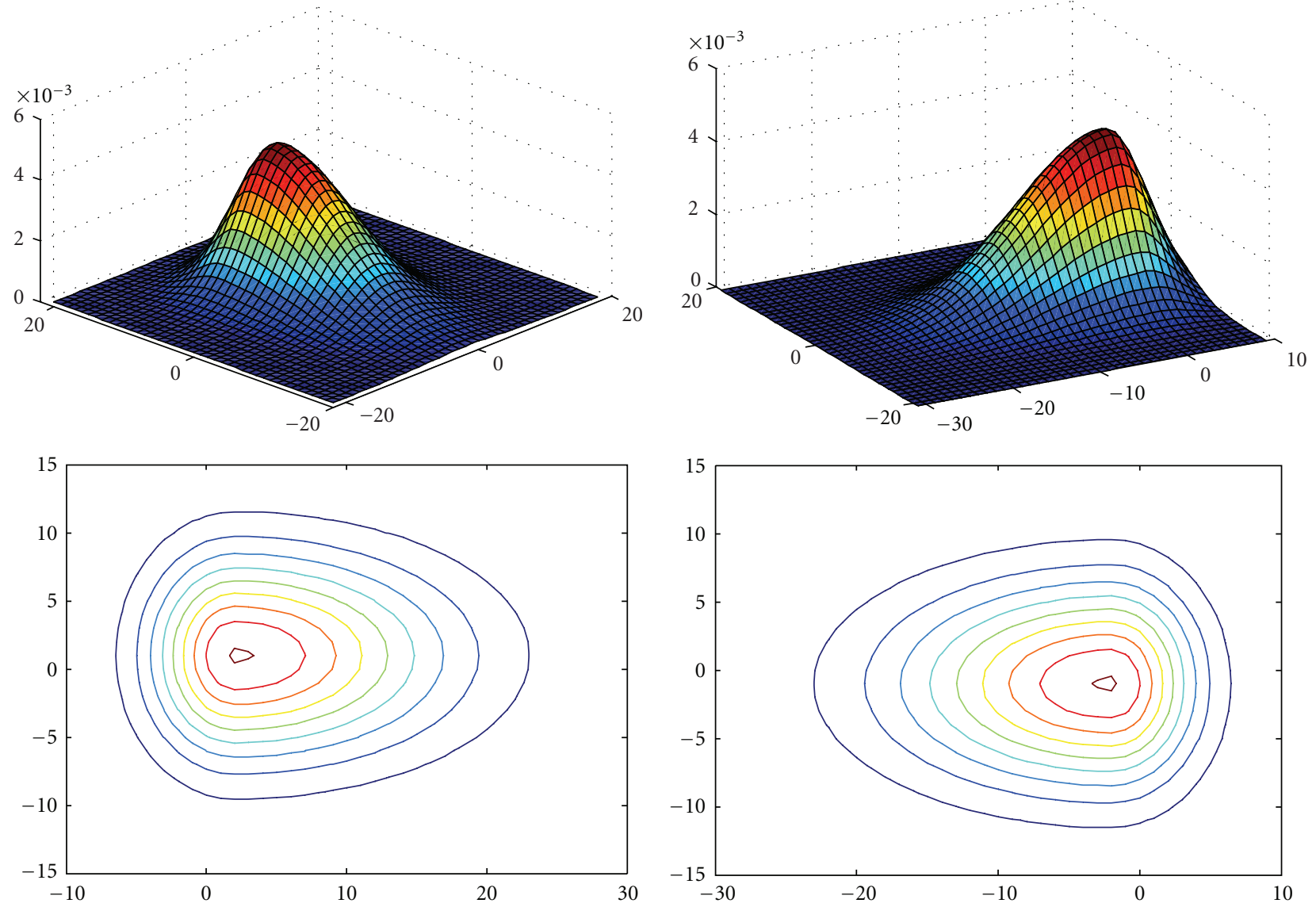

(a)

(b)

FIgURE 2: (a) Probability distribution of $f\left(x_{t}, y_{t}\right)$ for $m=7, \sigma_{y}=5, \sigma_{x_{1}}=10, \sigma_{x_{2}}=4$, (b) probability distribution of $f\left(x_{t}, y_{t}\right)$ for $m=7$, $\sigma_{y}=5, \sigma_{x_{1}}=4, \sigma_{x_{2}}=10$.

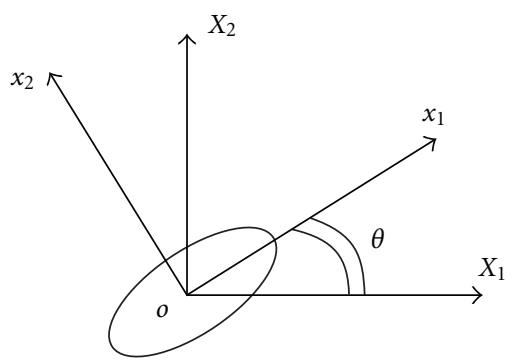

FIGURE 3: Elliptical gate in coordinate systems $x_{1} o x_{2}$ and $X_{1} O X_{2}$.

After some simplification, relations between parameters of elliptical gate and elements of innovation covariance could be achieved as follows:

$$
\begin{gathered}
\theta_{t}=\frac{1}{2} \operatorname{tg}^{-1}\left(\frac{2 S_{12, t}}{S_{11, t}-S_{22, t}}\right), \\
\sigma_{x, t}^{2}=-S_{11, t} \cos ^{2} \theta_{t-1}+S_{22, t} \sin ^{2} \theta_{t-1}, \\
\sigma_{y, t}^{2}=S_{11, t} \sin ^{2} \theta_{t-1}-S_{12, t} \sin ^{2} \theta_{t-1}+S_{22, t} \cos ^{2} \theta_{t-1},
\end{gathered}
$$

where $S_{11, t}, S_{12, t}, S_{21, t}, S_{21, t}$ and $S_{22, t}$ are elements of matrix $S_{t}$.

In this paper, Klaman filter is used to estimate parameters of asymmetric gate. The median that is achieved from Kalman filter is set as median of asymmetric Gaussian distribution which is the center of asymmetrical gate $m_{t}$. Using (14), $S_{11, t}, S_{12, t}, S_{21, t}, S_{21, t}$, and $S_{22, t}$ are used to estimate $\theta_{t}, \sigma_{x, t}$, and $\sigma_{y, t}$. It is assumed that $\sigma_{x_{1}, t}=\sigma_{x, t}$ as a standard deviation of asymmetrical gate on movement direction. To calculate parameter $\sigma_{x_{2}, t}$, estimated acceleration in movement direction, $\hat{a}_{x, t}$ is used which could be estimated from constant acceleration model and Kalman filter.

If $\hat{a}_{x, t}=0$, probability of existence of target in both side of median in movement direction are equal. Therefore, $\sigma_{x_{1}, t}$ is set equal to $\sigma_{x_{2}, t}$ and asymmetrical gate is converted to elliptical gate.

If $\hat{a}_{x, t}>0$, probability of target existence in movement direction is greater than negative side. It means that $\sigma_{x_{1}, t}$ should be greater than $\sigma_{x_{2}, t}$ which is resulted to following proposed equation:

$$
\frac{\sigma_{x_{1}, t}}{\sigma_{x_{2}, t}}=1+\frac{1}{2} \hat{a}_{x, t} T^{2},
$$


where $T$ is sampling time and $\hat{a}_{x, t}$ is estimation of acceleration in movement direction.

If $\hat{a}_{x, t}<0$, probability of existence of target in negative side of movement direction is greater than positive sight. So $\sigma_{x_{2}, t}$ should be greater than $\sigma_{x_{1}, t}$ which is resulted to following proposed equation:

$$
\frac{\sigma_{x_{2}, t}}{\sigma_{x_{1}, t}}=1-\frac{1}{2} \hat{a}_{x, t} T^{2} .
$$

In proposed estimation method, the ratio of standard deviations in direction of movement is proportional to sampling time and estimated acceleration in movement direction.

\section{Analytical Comparison between Asymmetrical and Elliptical Gate}

In this part, analytical comparison between asymmetrical gate and typical elliptical gate in tracking of targets with nonzero acceleration are studied. Volumes of gates are considered as a proper criterion in comparison. To compare volumes of gates, it is assumed that in-gate probabilities of distributions are equal for both gates.

In tracking with elliptical gate, probability of distribution in centric coordinate system is Gaussian as following:

$$
\begin{aligned}
f_{1}\left(z_{t}\right)= & \frac{1}{2 \pi \sigma_{x_{0}} \sigma_{y_{0}}} \\
& \times \exp \left(-\frac{1}{2}\left(z_{t}-m_{0}\right)^{T}\left[\begin{array}{cc}
\sigma_{x_{0}} & 0 \\
0 & \sigma_{y_{0}}
\end{array}\right]^{-1}\left(z_{t}-m_{0}\right)\right),
\end{aligned}
$$

where $m_{0}$ is median, $\sigma_{x_{0}}$ is standard deviation of distribution on movement direction, and $\sigma_{y_{0}}$ is standard deviation of distribution on orthogonal to movement direction.

In tracking with asymmetric gate, it is assumed that probability of target distribution in centric coordinate system is asymmetric gaussian as follows:

$$
\begin{aligned}
& f_{2}\left(z_{t}\right) \\
& =\frac{1}{2 \pi \sigma_{y_{1}}\left(\left(\sigma_{x_{1}}+\sigma_{x_{2}}\right) / 2\right)} \\
& \quad \times \exp \left(-\frac{1}{2}\left(z_{t}-m_{1}\right)^{T}\left[\begin{array}{cc}
\sigma_{x_{i}} & 0 \\
0 & \sigma_{y_{1}}
\end{array}\right]^{-1}\left(z_{t}-m_{1}\right)\right) \quad i=1,2,
\end{aligned}
$$

where $m_{1}$ is median, $\sigma_{x_{1}}$ and $\sigma_{x_{2}}$ are standard deviations of distribution on movement direction, and $\sigma_{y_{1}}$ is standard deviation of distribution on orthogonal to movement direction.

Because of the fact that probability distributions in (17) and (18) are represented in centric coordinate system, offdiagonal elements of covariance matrixes are zero.

To compare between asymmetric gate and elliptical gate, it is assumed that the target has positive acceleration in movement direction. Parameters of distributions (17) and
(18) could be estimated using Kalman filter and the proposed method in (15) and (16). Therefore, the relation between parameters of asymmetric gate and elliptical gate are achieved as follows:

$$
\begin{gathered}
\sigma_{x} \triangleq \sigma_{x_{0}}=\sigma_{x_{1}}, \\
\sigma_{y} \triangleq \sigma_{y_{0}}=\sigma_{y_{1}}, \\
m \triangleq m_{0}=m_{1}, \\
\sigma_{x_{2}}<\sigma_{x_{1}} .
\end{gathered}
$$

In-gate symmetric and asymmetric probability of distribution could be calculated as follows, respectively [14],

$$
\begin{aligned}
P_{1 G_{t}} & =\iint_{G_{t}} f_{1}\left(z_{t}\right) d x d y \\
& =\int_{0}^{d_{1} / 2} \exp (-u) d u=1-\exp \left(-\frac{d_{1}}{2}\right), \\
P_{2 G_{t}} & =\iint_{G_{t}} f_{2}\left(z_{t}\right) d x d y \\
& =\int_{0}^{d_{2} / 2} \exp (-u) d u=1-\exp \left(-\frac{d_{2}}{2}\right) .
\end{aligned}
$$

Symmetric gate volume $V_{1 G_{t}}$ and asymmetric gate volume $V_{2 G_{t}}$ are considered as follows:

$$
\begin{gathered}
V_{1 G_{t}}=d_{1} \pi \sigma_{y_{0}} \sigma_{x_{0}}, \\
V_{2 G_{t}}=d_{2} \pi \sigma_{y_{1}}\left(\frac{\sigma_{x_{1}}+\sigma_{x_{2}}}{2}\right) .
\end{gathered}
$$

It is assumed that, gate probabilities are equal in symmetric and asymmetric gate. Therefore, regarding (19), the following relation could be achieved:

$$
\left.\begin{array}{c}
\left\{P_{1 G_{t}}=P_{2 G_{t}} \Longrightarrow d_{1}=d_{2}\right\} \\
\sigma_{y_{0}}=\sigma_{y_{1}} \\
\left(\frac{\sigma_{x_{1}}+\sigma_{x_{2}}}{2}\right)<\sigma_{x_{0}}
\end{array}\right\} \Longrightarrow V_{2 G_{t}}<V_{1 G_{t}} .
$$

Using the above equation, we get that with equal in-gate probability for symmetric and asymmetric gates, volume of asymmetric gate would be smaller than volume of symmetric gate. It means that with equal probability for asymmetric and elliptical gate, computational complexity to find target inside asymmetrical gate would be less than computational complexity inside of elliptical gate. It is because of the fact that volume of asymmetrical gate is smaller than volume of elliptical gate with equal in-gate probability of distribution.

\section{Optimal Size of Gate}

Elliptical gates in [14] are represented as follows:

$$
\left(z_{t}-m_{z}\right)^{T} S_{t}^{-1}\left(z_{t}-m_{z}\right) \leq d,
$$

where $z_{t}=\left[\begin{array}{ll}x_{t} & y_{t}\end{array}\right], m_{z}$ is center of gate, and $S_{t}$ is innovation covariance matrix that has information about rotation direction of gate and ratio of diameters of gate. Asymmetrical gate that is proposed in this paper could be represented similar to $(23)$ as

$$
\left(z_{t}-m_{z}\right)^{T} S_{t}^{-1}\left(z_{t}-m_{z}\right) \leq d,
$$


where $z_{t}=\left[\begin{array}{ll}x_{t} & y_{t}\end{array}\right], m_{z}=\left[m_{t} 0\right]$, and

$$
S_{t}=\left[\begin{array}{cc}
\sigma_{x_{i}, t}^{2} & 0 \\
0 & \sigma_{y, t}^{2}
\end{array}\right]_{i \in\{1,2\}} .
$$

If $x_{t}>m_{t}$, we choose $i=1$ and if $x_{t}<m_{t}$, we choose $i=2$.

$z_{t}$ would be inside the gate if two following constraints are satisfied.

(a) If $x_{t}>m_{t}$, then

$$
\left(z_{t}-m_{z}\right)^{T}\left[\begin{array}{cc}
\sigma_{x_{1}, t}^{2} & 0 \\
0 & \sigma_{y, t}^{2}
\end{array}\right]^{-1}\left(z_{t}-m_{z}\right)<d .
$$

(b) If $x_{t}<m_{t}$, then

$$
\left(z_{t}-m_{z}\right)^{T}\left[\begin{array}{cc}
\sigma_{x_{2}, t}^{2} & 0 \\
0 & \sigma_{y, t}^{2}
\end{array}\right]^{-1}\left(z_{t}-m_{z}\right)<d .
$$

Otherwise, $z_{t}$ is out of the gate.

It is easy to see from (24) that shape of the gate could be determined by $m_{z}, \sigma_{x_{2}, t}^{2}, \sigma_{x_{1}, t}^{2}$, and $\sigma_{y, t}^{2}$, whereas the size of the gate could be smaller or larger using just $d$.

As the gate is made larger, more clutter or observation vectors other than the tracked target are included within it, so that tracking becomes more difficult. On the other hand, if the gate is made smaller, there is an increasing danger that the observation vector from the tracked target may no longer fall within the gate.

So, in this paper, we define a cost function to find optimum size of gate using the cost function for elliptical gates that is proposed in [14]. In this paper, using the method proposed in [14], optimum size of asymmetrical proposed gate is calculated.

Suppose that whole points inside of the gate are defined as follows:

$$
G_{t}=\left\{z_{t} \mid\left(z_{t}-m_{z}\right)^{T} S_{t}^{-1}\left(z_{t}-m_{z}\right) \leq d\right\} .
$$

In-gate probability could be calculated by:

$$
P_{G_{t}}=\iint_{G_{t}} f\left(x_{t}, y_{t}\right) d x d y,
$$

where $f\left(x_{t}, y_{t}\right)$ is asymmetrical Gaussian distribution.

After some simplifications, in-gate probability simplifies as follows:

$$
P_{G_{t}}=\int_{0}^{d / 2} e^{-u} d u
$$

From (30), it is visible that in-gate probability is independent from the shape of the gate which is defined using parameters $m_{z}, \sigma_{x_{2}, t}^{2}, \sigma_{x_{1}, t}^{2}$, and $\sigma_{y, t}^{2}$, and it is just function of the gate size $d$.

Volume of gate could be considered as

$$
V_{G_{t}}=\iint_{G_{t}} d x d y
$$

After simplification, volume of gate reduced to

$$
V_{G_{t}}=d \pi \sigma_{y, t-1}\left(\frac{\sigma_{x_{1}, t-1}+\sigma_{x_{2}, t-1}}{2}\right) .
$$

Assuming that probability of false alarm observation at time $t$ is a constant value $B_{f}^{t}$, mean number of false observed signals inside of the gate are $B_{f}^{t} V_{G_{t}}$ :

$$
B_{f}^{t} V_{G_{t}}=B_{f}^{t} d \pi \sigma_{y, t-1}\left(\frac{\sigma_{x_{1}, t-1}+\sigma_{x_{2}, t-1}}{2}\right) .
$$

With the assumption that target observation probability is constant $P_{D}$, the probability that target is observed inside of the gate is $P_{D} P_{G_{t}}$. In other side, mean number of false signals inside of the gate is $B_{f}^{t} V_{G_{t}}$. The goal is to find optimum size of the gate so that $P_{D} P_{G_{t}}$ is maximum and $B_{f}^{t} V_{G_{t}}$ is minimum. With respect to the fact that $P_{D} P_{G_{t}}$ and $B_{f}^{t} V_{G_{t}}$ are function of $d$, so it is possible to define $h(d)$ in such a way that, with maximizing it, optimum value of $d$ is calculated:

$$
\begin{gathered}
h(d)=P_{D} P_{G_{t}}-c B_{f}^{t} V_{G_{t}}, \\
h(d)=P_{D} \int_{0}^{d / 2} e^{-u} d u-c B_{f}^{t} d \pi \sigma_{y, t-1}\left(\frac{\sigma_{x_{1}, t-1}+\sigma_{x_{2}, t-1}}{2}\right),
\end{gathered}
$$

where $c$ is a constant value:

$$
\begin{aligned}
\frac{\partial h(d)}{\partial d}=0 \Longrightarrow d_{\mathrm{opt}}= & -2 \ln c-2 \ln 2 \pi-2 \ln B_{f}^{t} \\
& -2 \ln \left(\sigma_{y, t-1}\left(\frac{\sigma_{x_{1}, t-1}+\sigma_{x_{2}, t-1}}{2}\right)\right) \\
& +2 \ln P_{D} .
\end{aligned}
$$

Larger $c$ increase emphasis on $B_{f}^{t} V_{G_{t}}$ and number of false observed signals inside of the gate decreases.

\section{Simulation Result}

To simulate the preferences of the new method, in first we generate samples of a track using model (36). In this model, let $r_{x}(t)$ be position, let $s_{x}(t)$ be velocity, let $a_{x}(t)$ be acceleration of the target in movement direction, let $r_{y}(t)$ be position, let $s_{y}(t)$ be velocity, and let $a_{y}(t)$ be acceleration of the target in orthogonal to movement direction at time $t$ in centric coordinate system, where $t$ stands for continues time index. Dynamics of the target is described by system model that could be written in stochastic differential equation:

$$
\dot{X}(t)=A X(t)+B n(t),
$$

where $X(t)$ is state vector

$$
X(t)=\left[\begin{array}{llllll}
r_{x}(t) & s_{x}(t) & a_{x}(t) & r_{y}(t) & s_{y}(t) & a_{y}(t)
\end{array}\right]^{T} .
$$

$A$ is state transition matrix:

$$
A=\left[\begin{array}{llllll}
0 & 1 & 0 & 0 & 0 & 0 \\
0 & 0 & 1 & 0 & 0 & 0 \\
0 & 0 & 0 & 0 & 0 & 0 \\
0 & 0 & 0 & 0 & 1 & 0 \\
0 & 0 & 0 & 0 & 0 & 1 \\
0 & 0 & 0 & 0 & 0 & 0
\end{array}\right],
$$


vector $B$ is defined as

$$
B=\left[\begin{array}{llllll}
0 & 0 & 1 & 0 & 0 & 0 \\
0 & 0 & 0 & 0 & 0 & 1
\end{array}\right]^{T}
$$

vector $n(t)$ is defined as

$$
n(t)=\left[\begin{array}{ll}
n_{1}(t) & n_{2}(t)
\end{array}\right]^{T},
$$

where $n_{1}(t)$ is random noise with uniform distribution between zero and one and $n_{2}(t)$ is zero mean white gaussian noise.

By applying time discretization to (36) with sampling time $T$, we have a discrete time system model:

$$
X_{k}=A_{d} X_{k-1}+B_{d} n_{k}
$$

State transition matrix $A_{d}$ is

$$
A_{d}=\left[\begin{array}{cccccc}
1 & T & \frac{T^{2}}{2} & 0 & 0 & 0 \\
0 & 1 & T & 0 & 0 & 0 \\
0 & 0 & 1 & 0 & 0 & 0 \\
0 & 0 & 0 & 1 & T & \frac{T^{2}}{2} \\
0 & 0 & 0 & 0 & 1 & T \\
0 & 0 & 0 & 0 & 0 & 1
\end{array}\right],
$$

and vector $B_{d}$ is

$$
B_{d}=\left[\begin{array}{cccccc}
\frac{T^{3}}{3} & \frac{T^{2}}{2} & T & 0 & 0 & 0 \\
0 & 0 & 0 & \frac{T^{3}}{3} & \frac{T^{2}}{2} & T
\end{array}\right]^{T} .
$$

Measured position of the target in centric coordinate system is denoted by $z_{k}$ and is assumed to be obtained by observation model:

$$
z_{k}=C X_{k}+w_{k}
$$

where $C$ is

$$
C=\left[\begin{array}{llllll}
1 & 0 & 0 & 0 & 0 & 0 \\
0 & 0 & 0 & 1 & 0 & 0
\end{array}\right]
$$

and $w_{k}$ is defined as

$$
w_{k}=\left[\begin{array}{ll}
w_{1 k} & w_{2 k}
\end{array}\right]^{T},
$$

where $w_{1 k}$ is random noise with uniform distribution between zero and one and $w_{2 k}$ is zero mean white gaussian noise.

In Figure 4, target positions are shown that is generated using the above model. Monte Carlo simulation is used to approximate probability distribution of target position in next time step. Approximated probability of distribution is shown in Figure 4.

It is easy to understand from Figure 4 that probability distribution in next time could be illustrated using asymmetric distribution that formulated previously.

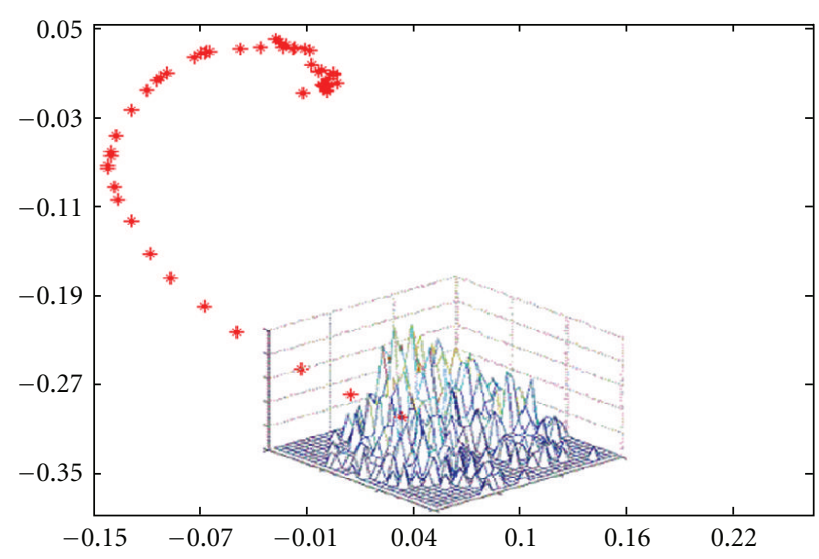

FIGURE 4: Target track and estimation of probability of distribution in next time step using Monte Carlo simulation.
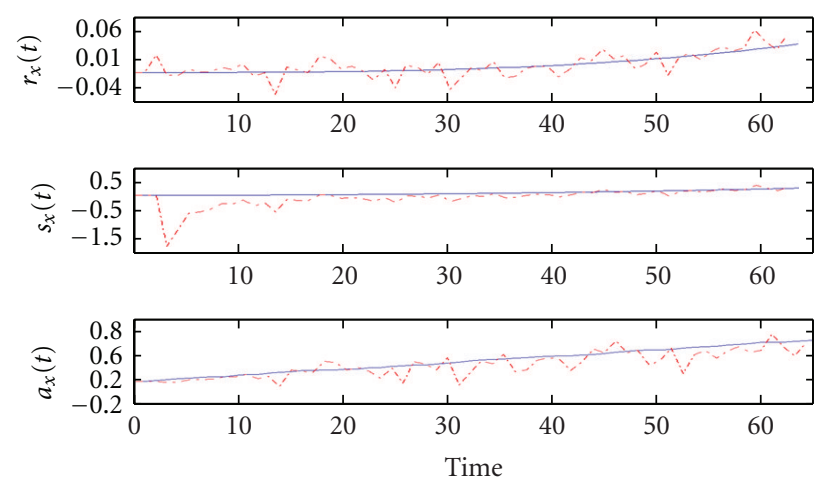

FIGURE 5: Actual (blue solid) and estimated (red dash) values of $r_{x}(t), s_{x}(t)$, and $a_{x}(t)$ in centric coordinate system.

Kalman filter is used to estimate parameters of asymmetric gate that are considered in part 3. Actual and estimated values of $r_{x}(t), s_{x}(t)$, and $a_{x}(t)$ in centric coordinate system are shown in Figure 5.

To show proficiency of the proposed asymmetrical gating method in comparison with previous gating methods such as circular gating, elliptical gating, and rectangular gating, similar simulation scenarios are studied for circular, elliptical, and rectangular gates. The volumes of gates in each simulation are equal and the location of the target in the next time step is important in the comparison between different gates. The number of times that the target falls in a gate in the next time step is a comparison index between gates. In Table 1, comparison results between circular gate, elliptical gate, rectangular gate, and proposed asymmetrical gate are shown for different gate volumes.

In Table 1, percent falls of the target inside each gate are shown for different volumes of gate. For example, for volume of gate 0.0025 , percent fall of the target inside rectangular gate is $51 \%$, inside circular gate is $65 \%$, inside elliptical gate is $73 \%$ and inside asymmetrical gate is $92 \%$.

For different volumes of gates, it could be seen from Table 1 that proposed asymmetrical gate has more in-gate probability than other gating methods and the number of 
TABLE 1: Comparison results between circular gate, elliptical gate, rectangular gate and asymmetrical gate for different gate volumes.

\begin{tabular}{lcccc}
\hline $\begin{array}{l}\text { Gate } \\
\text { volume }\end{array}$ & $\begin{array}{c}\text { Rectangular } \\
\text { gate }\end{array}$ & $\begin{array}{c}\text { Circular } \\
\text { gate }\end{array}$ & $\begin{array}{c}\text { Elliptical } \\
\text { gate }\end{array}$ & $\begin{array}{c}\text { Asymmetrical } \\
\text { gate }\end{array}$ \\
\hline 0.0025 & $51 \%$ & $65 \%$ & $73 \%$ & $92 \%$ \\
0.005 & $68 \%$ & $78 \%$ & $84 \%$ & $96 \%$ \\
0.01 & $90 \%$ & $98 \%$ & $100 \%$ & $100 \%$ \\
\hline
\end{tabular}

target falls inside of the asymmetrical gate in the next step is more than others. It is because of the asymmetrical gate ability to shape itself to have more percent falls of the target inside the gate. Although parameters of other gates can be updated in each step to have more percent falls inside the gates, the ability of shape changing in other gates is less than asymmetrical gate specially in maneuvering target tracking applications. Not that the percent falls of the target inside of the gates increases by increasing gate volume that could be seen easily from Table 1 .

\section{Conclusion and Future Remarks}

In this paper, we introduced asymmetric gating technique for tracking maneuvering targets that have nonzero acceleration in movement direction. Asymmetric gate is defined as a cross-surface of asymmetric Gaussian distribution. Standard Kalman filter is used to estimate some parameters of asymmetrical gate. Standard deviation of asymmetrical distribution in opposite to the movement direction is estimated using a proposed method in which sampling time and estimated acceleration are used. Preference of asymmetrical gate to the elliptical gate is proved analytically subject to constant in-gate probability of distribution for both gates, in which volumes of gates are used as a comparison criterion. We derived an optimization method to finding optimum size of asymmetrical gate. Optimum size of asymmetrical gate is obtained as a function of some parameters such as probability of target observation and probability of false alarms that should be tune manually in each special applications.

As a future remarks, it could be assumed that probability of distribution in orthogonal to the movement direction in centric coordinate system is considered asymmetric. Also, estimation of gate parameters could be studied in typical coordinate system, after enough research, and proves on generalization of Kalman filter for linear systems with asymmetric gaussian probability of distributions for both process and measurement noise.

\section{References}

[1] C. B. Chang and J. A. Tabaczynski, "Application of state estimation to target tracking," IEEE Transactions on Automatic Control, vol. 29, no. 2, pp. 98-109, 1984.

[2] Y. Bar-Shalom, "Tracking methods in a multi target environment," IEEE Transactions on Automatic Control, vol. 23, no. 4, pp. 618-626, 1978.
[3] X. R. Li and V. P. Jilkov, "A survey of maneuvering target tracking-part II: ballistic target models," in Signal and Data Processing of Small Targets, vol. 4473 of Proceedings of SPIE, pp. 559-581, 2001.

[4] D. C. Woffinden and D. K. Geller, "Observability criteria for angles-only navigation," IEEE Transactions on Aerospace and Electronic Systems, vol. 45, no. 3, pp. 1194-1208, 2009.

[5] Y. Bar-Shalom and E. Tse, "Tracking in a cluttered environment with probabilistic data association," Automatica, vol. 11, no. 5, pp. 451-460, 1975.

[6] T. E. Fortmann, Y. Bar-Shalom, and M. Scheffe, "Multitarget tracking using joint probabilistic data association," in Proceedings of IEEE Conference on Decision and Control, pp. 807-812, December 1980.

[7] D. B. Reid, "An algorithm for tracking multiple targets," IEEE Transactions on Automatic Control, vol. 24, no. 6, pp. 843-854, 1979.

[8] Y. Kosuge, S. Tsujimichi, and Y. Tachibana, "Track-oriented multiple hypothesis multi target tracking algorithm," IEICE Transactions, pp. 677-685, 1996.

[9] Y. Kosuge, S. Tsujimichi, S. Mano, and S. Betsudan, "Suboptimal techniques for track-oriented multiple hypothesis tracking algorithm and JPPA algorithm for multi target tracking to be equivalent," IEICE Transactions, pp. 889-898, 1997.

[10] S. S. Blackman, "Multiple Target Tracking with Radar Applications," p. Artech House, 1986.

[11] Y. Bar-Shalom and T. E. Fortman, Tracking and Data Association, Academic Press, New York, NY, USA, 1988.

[12] Y. Oshman, "An information fusion approach to missile guidance," in International Conference on Control, Automation and Systems (ICCAS '07), October 2007.

[13] E. M. Saad et al., "Filtered Gate Structure Applied to Joint Probabilistic Data Association Algorithm for Multi-Target Tracking in Dense Clutter Environment," International Journal of Computer Science, pp. 161-170, 2011.

[14] Y. Kosuge and T. Matsuzaki, "The gate size estimation method and the optimal gate shape for target tracking," Electronics and Communications in Japan, Part III, vol. 85, no. 5, pp. 10-22, 2002. 

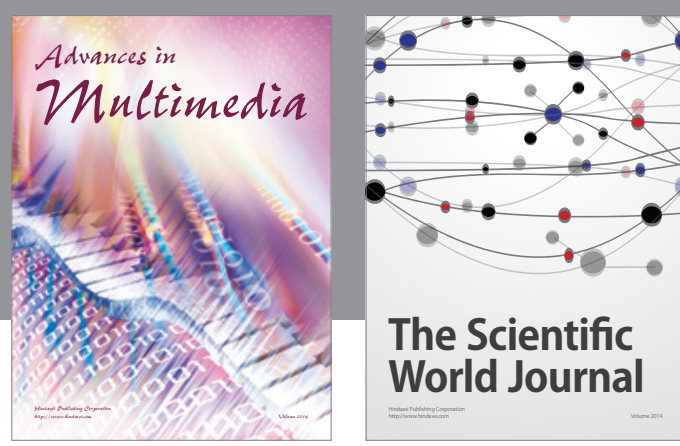

The Scientific World Journal
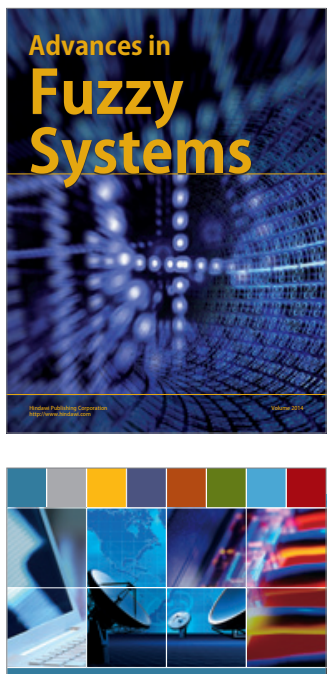

Computer Networks and Communications
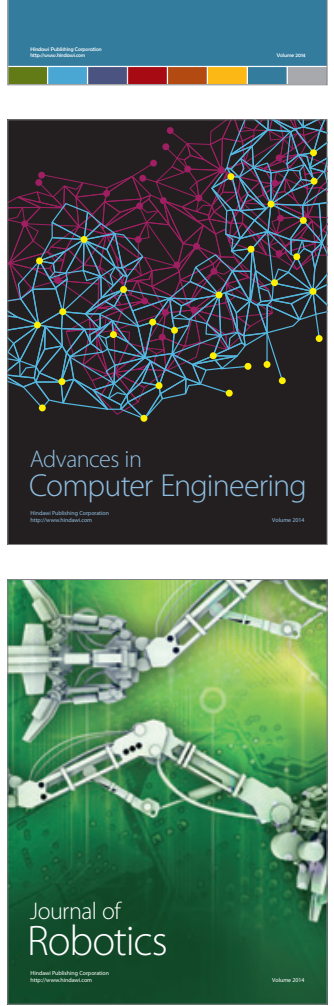
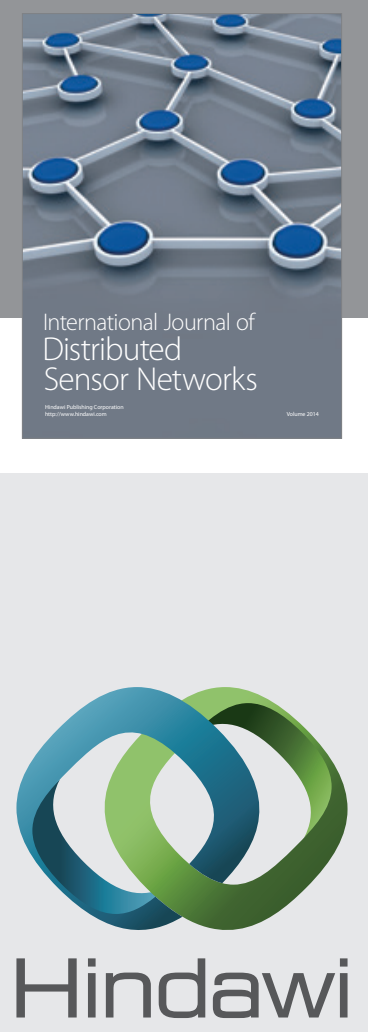

Submit your manuscripts at

http://www.hindawi.com
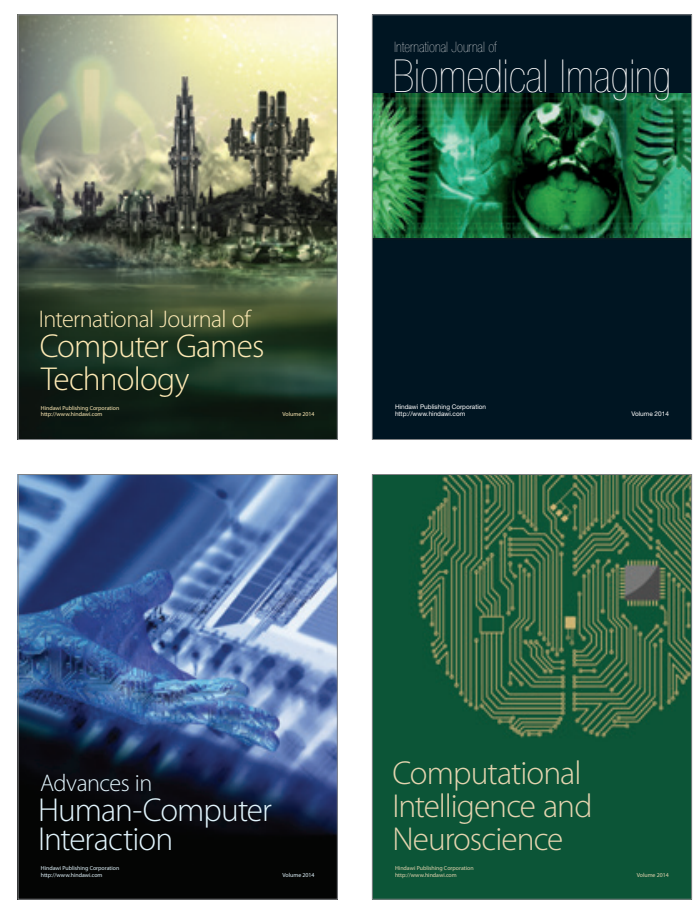
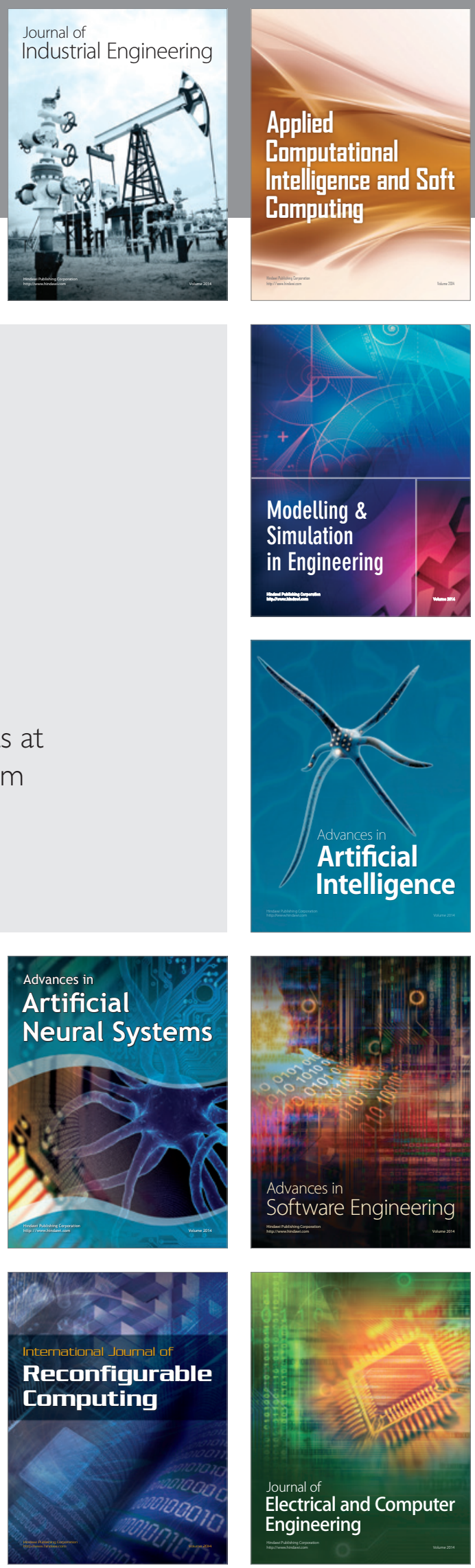